in 2017. Study of susceptibility of E. coli showed that resistant to antibiotics strains prevailed $(61.8 \%)$ and the ratio of such strains increased almost two-fold in 2017 (72.1\%) from 2016 (43.2\%). Most isolates were resistant to ampicillin and inhibitor-protected penicillins such as amoxicillin/ clavulanate $(61.0 \%)$. Proportion of such strains increased almost two-fold in 2017 (72.2\%) from 2016 (40.9\%). Resistant to fluoroquinolones, cephalosporins were 3.2\% and $2.4 \%$ strains, more than 20 times less. All of them were isolated in 2016. Isolates resistant to two preparations at the same time were most frequent $(58.5 \%)$. The proportion of multidrug resistant cultures was low (2.4\%). There were only 3 isolates resistant to ampicillin, inhibitor-protected penicillins, fluoroquinolones and cephalosporins.

Number of strains of diarrheagenic $E$. coli increased two-fold in 2017 compared with 2016, most of them were EIEC O144 and ETEC O25. Antibiotic-resistant cultures prevailed. Multidrug resistant strains were rare. All strains were susceptible to carbapenems, most of them - to fluoroquinolones and cephalosporins.

9.15 doi: 10.15789/2220-7619-2018-4-9.15

\section{MOLECULAR ANALYSIS OF PATHOGENS} OF PARTICULARLY DANGEROUS BACTERIAL INFECTIONS: FROM THEORY TO PRACTICE

\section{A.N. Kulichenko, D.A. Kovalev}

\section{Stavropol Plague Control Research Institute, Stavropol, Russia}

The modern period of development of medical and biological science is characterized by significant successes in the field of structural analysis of microorganisms and wide technological possibilities of obtaining living objects with given properties. A new scientific trend has emerged - synthetic biology. One of the urgent goals is the application of molecular methods in the practice of epidemiological analysis, to determine the source of infection and the pathways of the spread of the microbial pathogen, to assess its virulence and other properties.

At the present time, considerable material has been accumulated on the genetics of pathogens of anthrax, plague, cholera, brucellosis and other extremely dangerous microorganisms. The algorithms of PCR analysis have been developed in determining the epidemiological significance of isolate strains and their taxonomic features. There is experience of genotyping using MLVA, MLST, SNP and other methods, as well as the analysis of the complete genomic sequence (WGS).

Taking into account the levels of strain analysis (diagnosis of infection or epidemiological analysis), the following current research areas can be identified:

- Detection and identification: application of nucleic acid amplification methods for the differentiation of living and dead cells; introduction of multiplex (multifactor) PCR analysis technologies; creation (completion) of databases of mass spectra of microorganisms; introduction of methods of direct mass-spectrometric analysis of clinical material.

- Molecular typing: the creation of sequential (optimal) genotyping algorithms for each species; application of protein profiling methods for typing pathogens.

- Application of information systems, epidemiological analysis: creation of own databases of full-genomic sequencing; genomic profiling of pathogens in specific areas; creation of complex software products using the data of geographical information systems and predictive modeling.

As a result, an algorithm for bioinformational analysis should be developed for the epidemiological investigation of outbreaks (cases) of infectious diseases, including those caused by new (atypical) genetic variants of pathogens of especially dangerous infections.

DIFFERENTIATION OF KLEBSIELLA Spp. STRAINS FOR SENSITIVITY TO ANTIBIOTICS USING MASS SPECTROMETRY ANALYSIS MALDI-TOF

I.V. Likhachev ${ }^{1}$, E.V. Zueva ${ }^{1}$, E.S. Karpova ${ }^{1}$, E.S. Kunilova ${ }^{1}$, D.A. Dudko ${ }^{1}$, N.V. Mikhailov',2, S.A. Egorova' ${ }^{1}$, L.A. Kraeva ${ }^{1,2}$

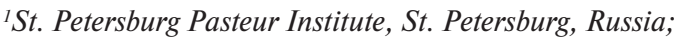
${ }^{2}$ S.M. Kirov Military Medical Academy, St. Petersburg, Russia

Klebsiella spp. strains are frequent causative agents of health care-related infections. Those strains especially dangerous if they circulate in hospitals. They are usually antibiotic-resistant. Therefore, information about the sensitivity of the isolated strain is necessary in the shortest time for proper etiological treatment.

The aim of our study was to assess the possibility of using mass spectrometry analysis (MALDI-TOF) for the rapid prediction of a selected Klebsiella spp. strain resistance.

The study used 195 strains of Klebsiella spp. isolated in various medical centers of St. Petersburg. All strains were identified by MALDI-TOF. Antibiotic sensitivity was studied by the disco-diffusion method in accordance with the recommendations of EUCAST 8.0. We used 19 antibiotics from 5 classes for testing strains: aminoglycosides, beta-lactams, beta-lactams of extended spectrum, quinolones and carbapenems.

A hierarchical clustering of spectra was made using the Unweighted Pair Group Method with Arithmetic Mean (UPGMA) to determine the relationship between clusters. We used Pearson correlation coefficient between variable values of peak intensity in spectral profiles as a measure of the distance between individual mass spectra. We identified 2 significant difference in the spectrum of the cluster. One cluster included spectra of strains resistant to all studied classes of antibiotics, second cluster strains sensitive to them (Distance Level - 0.65). It was also found that all strains included in the clusters, which differ from others by Distance Level more than 0.2, have the same profile of antibiotic resistance.

As a result of this work, we have formed profiles of phenotypic resistance of Klebsiella spp. strains to 19 antibiotics and 5 classes. The prospect of using the results of the study is a significant reduction in the study of biological material from the patient. Thus, simultaneously with the identification of Klebsiella spp. strains by MALDI-TOF it is possible to predict the sensitivity of the isolated strain to different classes of antibiotics or even to one of them. This will allow timely recognition of resistant strains of Klebsiella spp and prescribe adequate etiological therapy, which will significantly improve the quality of treatment of patients and will prevent the spread of resistant strains of bacteria in the medical establishments.

\subsection{7} doi: 10.15789/2220-7619-2018-4-9.17

\section{PATHOGENIC POTENTIAL OF COMMENSAL ESCHERICHIA COLI ISOLATED FROM ADULTS IN SAINT PETERSBURG}

\section{M.A. Makarova}

St. Petersburg Pasteur Institute, St. Petersburg, Russia

Escherichia coli is one constitute a component of the natural microbiota of warm-blooded animals including humans. At the same time commensal E.coli is a dynamic population and in some cases is capable to cause ex- 
traintestinal diseases, sometimes leading to morbidity and mortality. The aim of this work was to study of genetic diversity and assess of pathogenic potential of commensal E. coli isolated from healthy adults in Saint Petersburg. $300 E$. coli strains were collected from fecal samples of 50 St. Petersburg's inhabitants. E. coli strains were isolated using Endo agar and identified by biochemical tests. Determination of four major phylogenetic groups and identification of virulence genes were performed by using real-time, multiplex and simplex PCR. Seven genes typical for ExPEC (fimH, pap, sfa, aer, afa, $c n f 1, h l y A$ ) were identified among the analyzed strains. The B2 phylogroup $(47.1 \%)$ was leading among other groups: A $(20.5 \%), \mathrm{B} 1$ $(9.0 \%)$ and D (23.4\%). Each strain had at least one virulence gene. No strain had all seven studied genes simultaneously. The maximum number of genes in one strain was five. The prevalence of virulence genes was as follows: fim $H$ (98.0\%), pap (25.0\%), sfa (8.0\%), aer $(33.8 \%)$, afa $(5.6 \%)$, cnf1 (11.0\%), hlyA (10.0\%). The strains of groups B2 and $\mathrm{D}$ harbored the virulence determinants significantly more frequently then the strains of groups A and B1. Our results showed that $E$. coli isolated from adults differ in their phylogenetic structure and harbour a greater variety of virulence genes. Our study revealed that commensal $E$. coli isolated from healthy humans constitute a substantial reservoir of genes related to the extraintestinal pathotypes. All seven tested virulence genes typical for ExPEC were detected and it's important that the prevalence of these genes was significantly higher among the isolates from healthy adults. So, the extraintestinal virulence genes (encoding the adhesins, toxins, persistence) were found not only in pathogens, but also in commensal microflora of healthy people. Previous reports indicated that virulence genes associated with extraintestinal pathogenesis in fact help the $E$. coli strains to colonize the human gut; therefore, they may be considered as a fitness factor and the virulence is a coincidental side effect.

\subsection{8 \\ doi: 10.15789/2220-7619-2018-4-9.18 \\ EXPRESS METHOD OF GROWING BACTERIA ON THE MEMBRANE OF ANODIC ALUMINIUM OXIDE}

\section{N.I. Mukhurov ${ }^{1}$, L.A. Kraeva ${ }^{2,3}$, G.N. Khamdulaeva ${ }^{2}$}

${ }^{1}$ State Scientific and Production Association "Optic, Optoelectronic and Laser Technique”, St. Petersburg, Russia; ${ }^{2}$ St. Petersburg Pasteur Institute, St. Petersburg, Russia: ${ }^{3}$ S. M. Kirov Military Medical Academy, St. Petersburg, Russia

The classical method of studying bacteria is the cultivation of microorganisms and study of their biological properties. However, this method is very long (several days). So it may not be used in cases when you need to quickly get the result. It can be surgery, sepsis, severe infection, etc. In these cases, the doctor will need a few hours to make a decision on the appointment of causal treatment.

We have developed a method of growing the isolated clones of bacteria from any biological material for 3 hours. The rapid growth of microorganisms is ensured due to the new culture medium. Each microbial cell is grown in a separate cell on a porous membrane of anodic aluminum oxide. After 3 hours of incubation reads visual information using a specially developed image sensor zoom. The visual image of the individual microcolony identified to the species created by special computer programs. The probability of coincidence of the results is $90 \%$. With the help of a special counter counts the number of bacteria of each species in the studied sample. This is especially important in the study of biological material containing several types of microorganisms.
Thus, 3 hours after inoculation of biological material, we get the result about of species and quantitative composition of bacteria. A living culture of microorganisms can work with it further to explore other biological properties, including rapid determination of sensitivity to antibiotics.

9.19 doi: 10.15789/2220-7619-2018-4-9.19

\section{THE DRUG RESISTANCE MUTATIONS} OF THE HEPATITIS B VIRUS AMONG HIV-INFECTED INDIVIDUALS

Yu.V. Ostankova

St. Petersburg Pasteur Institute, St. Petersburg, Russia

Hepatitis B virus (HBV) is one of the most common hepatotropic viruses that can cause both acute and chronic course of the disease. One form of chronic viral hepatitis $\mathrm{B}$ is occult hepatitis $\mathrm{B}$, characterized by the presence of HBV DNA in the liver and undetectable levels of HBsAg and HBV DNA in the peripheral blood. The co-infection of HBV with the human immunodeficiency virus (HIV) is facilitated by the common mechanisms and pathways of infection. Although the effect of HBV on the progression of HIV infection appears to be minimal, HIV affects the progression of liver fibrosis, increasing the risk of developing hepatocellular carcinoma and cirrhosis. The need for timely identification $\mathrm{HBV}$ variants carrying drug resistance mutations among $\mathrm{HBV} / \mathrm{HIV}$-coinfected patients.

The aim of our study was to evaluate the prevalence of $\mathrm{HBV}$ with drug resistance mutations among $\mathrm{HBV} / \mathrm{HIV}$ coinfected patients.

The material was blood plasma of 264 HIV-infected (HBsAg-) patients with virologic ineffectiveness of ARVT. A method for detecting HBV DNA with a low viral load based on a two-step PCR, followed by sequencing was used.

HBV DNA was detected in 89 (33.7\%) patients. Based on the phylogenetic analysis it was shown that in this group the HBV subgenotypes are represented in the following ratios: D1 - 39.3\%, D2 - 29.2\%, D3 - 30.4\%, C1 - 1.1\%, respectively. In the analysis of nucleotide sequences in the viral polymerase reverse transcriptase domain significant amino acid substitutions (mutations described in the literature as determining the development of drug resistance to nucleotide/nucleoside analogues therapy) were found in $12.35 \%$ of patients. Including 9 patient was found to have significant amino-acid replacement in HBV polymerase gene (L180M, M204V) associated with the development of resistance to lamivudine, entecavir, telbivudine and tenofovir. Also in $5.6 \%$ of patients were found potentially significant (substitutions in the same significant positions of the polymerase gene, but not described in the literature) - for example L80F.

The obtained data on the prevalence of HBV drug resistance indicate the need for screening of patients with HBV/ HIV-coinfection before starting the antiviral therapy.

\subsection{0}

doi: 10.15789/2220-7619-2018-4-9.20

INCREASE OF SELECTIVE AND GROWTH PROPERTIES OF A NUTRIENT MEDIUM FOR IDENTIFICATION AND ACCUMULATION TRICHOMONAS VAGINALIS

Y.V. Petrova ${ }^{1}$, O.V. Zarucheynova ${ }^{1}$, E.L. Saveleva ${ }^{1}$, N.A. Fedosyuk ${ }^{1}$, A.A. Samoilova ${ }^{2}$, V.N. Verbov ${ }^{1}$

${ }^{1}$ St. Petersburg Pasteur Institute, St. Petersburg, Russia; ${ }^{2}$ State Institute of Technology, St. Petersburg, Russia

Currently, the detection of patients with trichomoniasis is decreasing. This effect can be caused by asymptomatic infection, imperfect methods of protozoa identification, low availability and quality of nutrient media. Therefore, 